
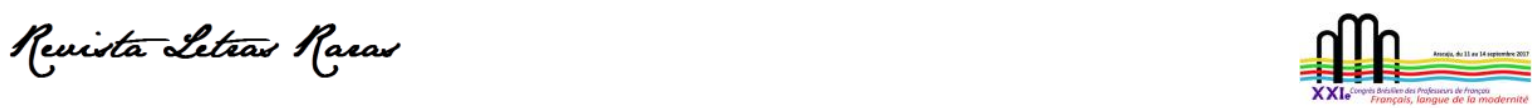

Actes du XXlème Congrès Brésilien des Professeurs De Français dans Édition spéciale de la Revue Letras Raras, 2021

ISSN : 2317-2347 - v. 10, Dossier Spécial (2021)

Todo o conteúdo da RLR está licenciado sob Creative Commons Atribuição 4.0 Internacional

\title{
Le professeur de DdNL et sa formation
}

\section{Fernanda Cristina Puça França*}

Docteure en Éducation (Formation de professeurs) de l'Université Fédérale de Pernambuco - UFPE (stage doctorale à l'Université Toulouse Jean Jaurès, en France) et professeure de FLE au Colégio de Aplicação de l'UFPE (CAp/UFPE).

D https://orcid.org/0000-0001-9199-1097

Reçu en : 28 juin 2021. Approuvé en: 20 juillet 2021.

\section{Comment citer cet article:}

FRANÇA, Fernanda Cristina Puça. Le professeur de DdNL et sa formation. Revista Letras Raras, p. 194205, n. Spécial, v. 10, nov. 2021.

\section{RÉSUMÉ}

L'enseignement d'une Discipline dite Non Linguistique (DdNL) en langue étrangère (LE) engage des connaissances disciplinaires et met en œuvre deux langues pour la construction et la transmission des connaissances. Le professeur de DdNL joue un rôle central et spécifique dans un enseignement bilingue. Selon Duverger (2009), sans professeur de DdNL, il n'y a pas d'enseignement bilingue. Cet article propose de décrire l'enseignement d'une Discipline dite Non Linguistique en langue française et de présenter les caractéristiques du professeur responsable pour ce travail et sa formation. II ne prétend pas épuiser le thème, mais soulève des aspects qui méritent réflexion et qui devraient être pris en compte dans l'organisation d'une formation initiale et/ou permanente des professeurs de DdNL.

MOTS-CLÉS: Bi/plurilinguisme ; professeur de Discipline dite Non Linguistique (DdNL) ; formation de professeur de DdNL.

\section{Introduction}

Nos sociétés sont de plus en plus marquées par le bi/plurilinguisme et la diversité culturelle. Le bi/plurilinguisme, par exemple, fait déjà partie de la société de nos jours. Dans un enseignement bilingue, deux ordres de disciplines sont mis en contact : les disciplines dites « linguistiques »(DL) et les disciplines dites non linguistiques (dorénavant DdNL). La première véhicule l'enseignement et la deuxième apporte les contenus cognitifs de cet enseignement. Toutes les DdNL passent par les $\mathrm{DL}$ pour se construire et se transmettre. L'enseignement d'une DdNL en langue étrangère (LE)

\section{\ernandapuca@capufpe.com}



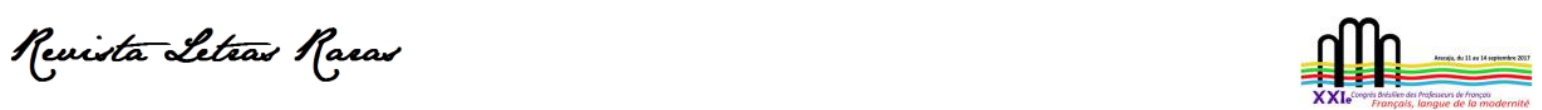

Actes du XXlème Congrès Brésilien des Professeurs De Français dans Édition spéciale de la Revue Letras Raras, 2021 ISSN : 2317-2347 - v. 10, Dossier Spécial (2021)

Todo o conteúdo da RLR está licenciado sob Creative Commons Atribuição 4.0 Internacional

engage des connaissances disciplinaires et met en œuvre deux langues pour la construction et la transmission des connaissances.

Dans le travail avec une DNL (Discipline Non Linguistique) ou DdNL (Discipline dite Non Linguistique) ou DEL2 (Discipline enseignée en langue 2) la langue n'est plus une fin, mais un outil pour accéder à des connaissances. Les deux langues, la langue maternelle (LM) et la langue étrangère (LE) doivent coexister dans le même cours d'une manière naturelle. La conception pour l'enseignement bilingue, la DdNL en langue française, est celle d'un enseignement qui utilise les deux langues en classe (DUVERGER, 2009), en opposition à une immersion totale (comme dans un cours de FLE - Français Langue Étrangère). Un travail sur la langue directement relié au contenu et un professeur qui utilise des formes et des degrés d'alternance entre les langues (GAJO, 2001).

Le professeur d'une DdNL est un professeur d'une matière scolaire qui enseigne en deux langues. Son objectif premier est d'aider les apprenants à s'approprier les connaissances et les concepts de sa matière. D'après Coste (2003), la langue étrangère est importante, mais l'emphase ne doit pas être mise sur la langue elle-même. Le professeur doit fixer son attention sur le contenu de la matière enseignée. II y a un gain linguistique, bien sûr, mais il doit être secondaire. Le professeur de DdNL doit mettre en relation pour chaque thématique, les objectifs, les contenus, les méthodologies, les stratégies d'apprentissage. Les objectifs pour les élèves sont l'enrichissement cognitif, l'ouverture vers une nouvelle perspective d'apprentissage et les différents savoirs qui pourront être approfondis en classe.

Une bonne formation linguistique pour un professeur de DdNL est nécessaire tout comme la construction des compétences linguistiques propres à sa discipline, c'est-à-dire, une sorte de français de spécialité (le lexique, les types de discours, la langue et ses variations et les alternances codiques de reformulation, par exemple). La connaissance linguistique de la langue étrangère est importante, mais elle n'est pas la seule à être développée dans la formation d'un professeur de DdNL.

Cet article vise à décrire l'enseignement d'une Discipline dite Non Linguistique en langue française et de présenter les caractéristiques du professeur responsable pour ce travail et sa formation. II ne prétend pas épuiser le thème, mais soulève des aspects qui méritent réflexion et qui devraient être pris en compte dans l'organisation d'une formation initiale et/ou permanente des professeurs de DdNL. 

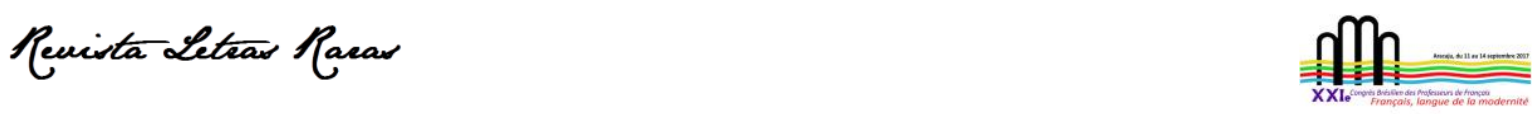

Actes du XXlème Congrès Brésilien des Professeurs De Français dans Édition spéciale de la Revue Letras Raras, 2021 ISSN : 2317-2347 - v. 10, Dossier Spécial (2021)

Todo o conteúdo da RLR está licenciado sob Creative Commons Atribuição 4.0 Internacional

\section{L'enseignement d'une Discipline dite Non Linguistique (DdNL)}

« [...] enseignement bi-/plurilingue [...] un enseignement engageant avant tout des connaissances disciplinaires et mettant en œuvre à cette fin deux langues (ou plus) pour la construction et la transmission de connaissances : la langue de scolarisation principale et la(les) langue(s) seconde(s) ou étrangère(s) ». (COSTE, 2003, p.02)

L'enseignement et l'apprentissage des langues étrangères ne se font pas uniquement dans des domaines spécifiques dans lesquels ils sont explicitement définis et réservés. Une Discipline dite Non Linguistique enseignée en langue française est une expérience bilingue. Selon Vollmer (2006), cet apprentissage est également présent dans toutes les autres matières scolaires et dans les activités scolaires et mentales, que nous soyons ou pas au courant de ce processus. L'élève et le professeur interagissent en classe et utilisent le français et le portugais comme des langues pour apprendre, enseigner et partager. Selon Duverger (2009), l'éducation est considérée comme bilingue lorsque les deux langues sont officiellement et structurellement présentes dans le système scolaire, pour la communication et, surtout, pour l'apprentissage. Le principe est de garantir la parité dans l'utilisation des langues dans les cours. Cette méthodologie n'est pas récente, puisqu'à partir du 19ème siècle il y a enseignement bilingue à Malte et plurilingue au Luxembourg (CUQ ; GRUCA, 2017).

La DdNL est une forme d'apprentissage combinant des aspects linguistiques et disciplinaires. Par l'utilisation d'une langue vivante étrangère (ou langue régionale ou langue minoritaire, avec un statut officiel), l'enseignement de disciplines dites non linguistiques a donc un double objectif : permettre à l'élève, d'une part, d'acquérir des connaissances dans les contenus spécifiques du programme d'études et, d'autre part, de développer des compétences linguistiques dans la langue étrangère. Ce sont deux langues utilisées et d'après Duverger (2009) l'utilisation régulière de la $L E$ dans un contexte de communication authentique aide indéniablement les élèves à progresser dans leur maîtrise de cette langue. Pour l'auteur, les élèves développent des compétences métalinguistiques qui leur permettent de mieux comprendre le fonctionnement de leur langue maternelle. L'auteur insiste sur le fait que l'utilisation d'une LE à l'école pour communiquer et pour apprendre augmente la connaissance de la manière qu'a l'autre d'appréhender la réalité et ajoute que l'enseignement bilingue permet d'accroître considérablement 

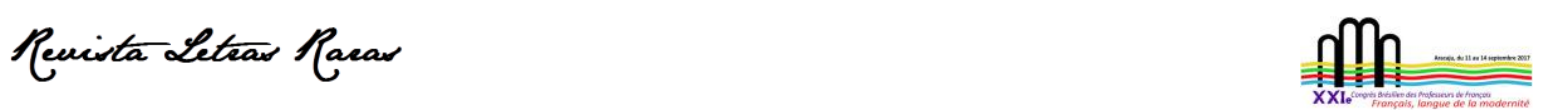

Actes du XXlème Congrès Brésilien des Professeurs De Français dans Édition spéciale de la Revue Letras Raras, 2021 ISSN : 2317-2347 - v. 10, Dossier Spécial (2021)

Todo o conteúdo da RLR está licenciado sob Creative Commons Atribuição 4.0 Internacional

l'ouverture culturelle de l'élève au monde, ainsi que sa tolérance aux différences. II faut transmettre à l'élève les valeurs de tolérance et de respect des autres cultures et le préparer à une société internationalisée. Pour Duverger (2009), les DdNL motivent l'apprentissage des langues, développent différentes compétences transversales et méthodologiques, permettent l'emploi de registres de langue spécifiques aux disciplines (tant au niveau lexical que syntaxique), aiguisent la curiosité, mais aussi les capacités d'attention et d'écoute des élèves.

Coste (2005) soutient que l'enseignement bilingue est une des voies de renouvellement de l'enseignement des langues dans les systèmes éducatifs, mais il faut qu'il soit inscrit dans un projet curriculaire institutionnel plus global. Lambert (1960) ajoute que les études montrent des avantages pour les élèves bilingues. Une flexibilité cognitive est observée chez les enfants qui ont I'habitude de passer d'un système de symboles à l'autre. Ils ont une plus grande capacité d'abstraction et cela potentialise la construction de concepts et la résolution de problèmes abstraits. Ils perçoivent qu'il y a plus d'une façon de s'exprimer dans différentes situations, ils trouvent facilement des réponses différentes à une question ou à un problème, ils sont créatifs et ouverts à d'autres cultures.

Les avantages de l'approche DdNL sont nombreux, à bien des égards. II y a les avantages linguistiques, culturels et socio-économiques (DUVERGER, 2009). L'utilisation d'une langue étrangère comme outil pour apprendre d'autres matières scolaires montre que la LE devient active, fonctionnelle et nécessaire. La langue modifie son statut et devient progressivement un outil d'apprentissage. Cela signifie que l'élève doit comprendre en LE, s'il veut accéder au contenu des différentes matières scolaires (les matières qui proposent l'approche DdNL). Apprendre une langue étrangère n'est pas un acte passif, son apprentissage aide à apprendre d'autres choses. L'élève apprend une LE quand il l'utilise dans différents contextes. Apprendre une langue étrangère est bénéfique pour le développement des compétences cognitives. Les avantages sont en termes de compétences transversales, d'approches méthodologiques et de concepts. L'apprentissage dans deux langues développe des stratégies et des méthodes d'apprentissage, favorise les constructions conceptuelles de chaque discipline car ces disciplines sont abordées dans les deux langues avec des exemples et des approches différents. Chaque langue, chaque culture pédagogique et chaque école liée à cette langue propose pour chaque discipline des approches méthodologiques spécifiques. Les élèves apprennent de manière plus naturelle, sans se limiter 

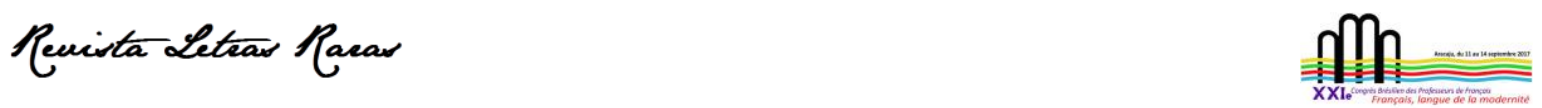

Actes du XXlème Congrès Brésilien des Professeurs De Français dans Édition spéciale de la Revue Letras Raras, 2021 ISSN : 2317-2347 - v. 10, Dossier Spécial (2021)

Todo o conteúdo da RLR está licenciado sob Creative Commons Atribuição 4.0 Internacional

aux frontières d'une seule discipline et peuvent prendre l'habitude d'être véritablement acteurs de leur apprentissage.

La perspective de l'enseignement bilingue est un pas vers une éducation plurilingue. L'importance du plurilinguisme comme activité sociale et cognitive est de plus en plus reconnue: du répertoire linguistique utilisé dans l'apprentissage autant que la stratégie plurilingue et des aspects positifs et négatifs qui obligent les institutions et les enseignants à prendre en compte cette réalité vivante sont présents à nos jours. L'enseignement d'une DdNL n'existe pas pour remplacer l'enseignement d'une langue étrangère ou l'enseignement en langue maternelle, mais pour augmenter les possibilités d'apprentissage par l'élève et son insertion dans ce monde globalisé dans lequel nous nous trouvons.

\section{Le professeur de DdNL}

Pour chaque langue que nous parlons, nous vivons une nouvelle vie. Quiconque ne connaît qu'une seule langue ne vit qu'une fois. (Proverbe tchèque)

Duverger (2009) atteste que le professeur de DdNL joue un rôle central et spécifique dans un enseignement bilingue. Sans professeur de DdNL, il n'y a pas d'enseignement bilingue. II utilise deux langues en classe et fait un enseignement singulier et spécialisé. Les élèves doivent utiliser la langue étrangère de manière naturelle dans le quotidien scolaire, durant les cours de biologie, d'arts, de mathématiques, où l'élève fabrique la plus value, ajoute du sens, de la motivation, de l'attention, car ces disciplines font partie du travail scolaire de l'apprenant.

Dans un contexte européen, le professeur de DdNL est encore rare et son statut institutionnel n'est pas encore stabilisé. Les professeurs de DdNL ne sont pas nombreux et leurs fonctions ne sont pas bien définies. II doit être clair que leur rôle n'est pas le renforcement de l'enseignement de la langue étrangère, ce n'est pas une aide destinée à remédier aux carences en matière d'enseignement d'une langue étrangère dans un établissement scolaire. Le professeur de DdNL n'est pas un professeur de langue étrangère et son but n'est pas le travail de la langue étrangère mais plutôt le travail en langue étrangère. II est le spécialiste de sa discipline 

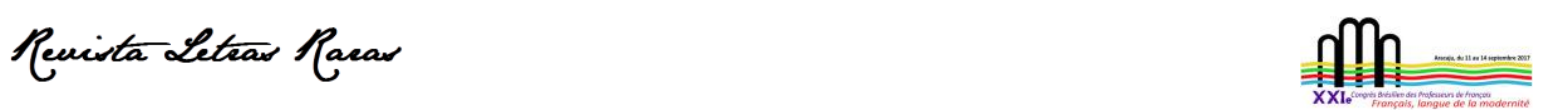

Actes du XXlème Congrès Brésilien des Professeurs De Français dans Édition spéciale de la Revue Letras Raras, 2021 ISSN : 2317-2347 - v. 10, Dossier Spécial (2021)

Todo o conteúdo da RLR está licenciado sob Creative Commons Atribuição 4.0 Internacional

d'enseignement. II faut qu'il maîtrise bien la LE pour qu'il soit suffisamment à l'aise dans son utilisation. Néanmoins, enseigner une DdNL en LE n'est pas exclusivement une question de maîtrise du système linguistique de la LE (comme nous l'avons déjà observé).

Le professeur de DdNL n'est pas un professeur de langue étrangère, mais il doit reconnaître la diversité et l'interculturalité à partir des différences d'enseignement, de choix de documents pour travailler dans les cours, de travail - soit par groupes, soit en binôme. D'après Duverger (2010), le professeur qui travaille dans une perspective bilingue doit s'efforcer pour construire un nouveau cours. Ce cours doit être original, singulier, bilingue, il doit mettre en relation des programmes, croiser des méthodologies et mettre en pratique l'alternance régulière des langues. Ce cours doit être conçu dans la langue étrangère et il ne doit pas être la traduction d'un cours en langue maternelle. Les langues doivent exprimer des concepts, des cultures et des moyens de les communiquer. Tout cela pour travailler des objectifs, d'abord disciplinaires, mais aussi linguistiques et culturels. Et cela se fait à partir d'une imbrication disciplinaire, vers une didactique intégrée des langues et des disciplines. L'apprenant devra parler maths, histoire, biologie, ... avec les moyens linguistiques dont il dispose et produire des textes (à l'oral comme à l'écrit) en adéquation avec les principes du discours des mathématiques, de l'histoire, de la biologie et des autres matières scolaires.

Pour cela il faut « la connaissance et l'appropriation des différents types de discours et de leur organisation en fonction des paramètres de la situation de communication dans laquelle ils sont produits et interprétés. » (MOIRAND 1982 : 20). Le Cadre Européen Commun de Référence pour les Langues - CECR (CONSEIL DE L'EUROPE, 2005) révèle que la compétence discursive permet à l'utilisateur/apprenant d'ordonner les phrases en séquences afin de produire des ensembles cohérents. L'enjeu est de développer des compétences plurilingues et pluriculturelles pour construire des références collectives qui promeuvent la diversité linguistique et culturelle afin que les professeurs puissent développer leur personnalité sociale avec et à travers différentes langues et cultures. Le professeur de DdNL doit constamment réfléchir aux cours qu'il planifie et donne aux élèves et à comment aider ses élèves à comprendre et à appliquer ce qui se construit en classe. 

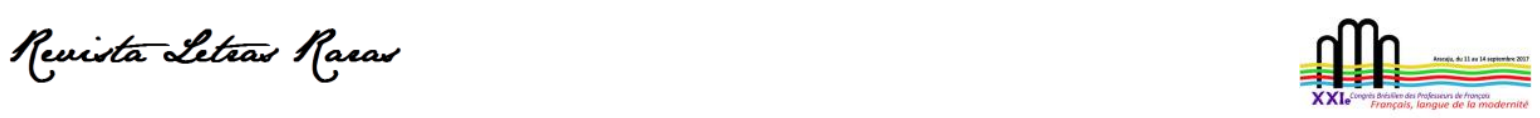

Actes du XXlème Congrès Brésilien des Professeurs De Français dans Édition spéciale de la Revue Letras Raras, 2021 ISSN : 2317-2347 - v. 10, Dossier Spécial (2021)

Todo o conteúdo da RLR está licenciado sob Creative Commons Atribuição 4.0 Internacional

\section{La formation du professeur de DdNL}

La formation des professeurs contribue à la création des individus autonomes, critiques et engagés pour la démocratie et la justice sociale. En tant que chercheurs en action, les professeurs visent la transformation de l'éducation, le renforcement du pouvoir individuel et collectif, autrement dit, une société plus large. La connaissance quotidienne et la réflexion en action, les interruptions devant les questions des élèves, impliquent un arrêt, permettant au professeur de se transformer en un chercheur-en-action produisant des connaissances fondamentales issues de l'engagement de l'investigation réflexive de leur propre pratique. Pour Zabala (1998), toute proposition méthodologique présuppose une conception de la valeur attribuée à l'enseignement.

La formation d'un professeur de DdNL doit fournir des outils pour que le professeur puisse construire une compétence discursive. Les différents types de discours : narratifs, descriptifs, argumentatifs, informatifs, explicatifs et injonctifs sont toujours disponibles aux enseignants de DdNL et sont définis selon l'intention de celui qui parle ou écrit. II y a une écriture et une cohérence didactique de séquences en DdNL, pour cela il faut travailler, en même temps, les ressources techniques et pédagogiques. L'objectif principal de l'enseignement intégré langue - discipline est la mise en place d'une compétence discursive intégrée.

La construction d'une compétence discursive semble être le point nodal du travail conjoint entre le professeur de FLE et le professeur de DdNL. Dans une formation permanente de DdNL, il faut fournir des outils pour que les professeurs puissent gérer les alternances codiques, construire des séquences pédagogiques, développer un travail collaboratif avec le professeur de FLE, construire une compétence discursive et maîtriser les discours des disciplines. La macroalternance linguistique est une répartition au sein du programme de la DdNL entre les chapitres/dossiers/sujets qui seront traités en LM ou en LE. La micro-alternance se situe au sein des séances de $\mathrm{DdNL} /$ séquences pédagogiques pendant lesquelles le professeur peut alterner les deux langues selon le niveau des élèves et selon des critères de progression disciplinaire. Duverger (2009) assure que la formation se doit donc de mettre l'accent sur les compétences linguistiques particulières propres à chaque discipline. Elle a pour objectif d'ordonner et de clarifier les rythmes d'alternance et permet de résoudre les problèmes de surcharge d'emploi du temps. 

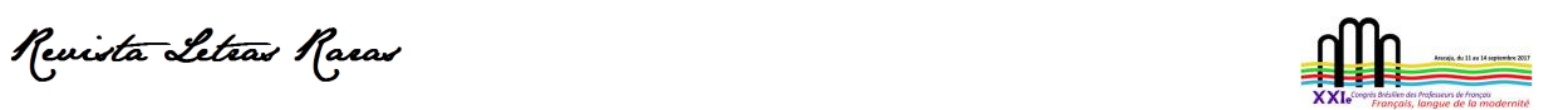

Actes du XXlème Congrès Brésilien des Professeurs De Français dans Édition spéciale de la Revue Letras Raras, 2021 ISSN : 2317-2347 - v. 10, Dossier Spécial (2021)

Todo o conteúdo da RLR está licenciado sob Creative Commons Atribuição 4.0 Internacional

L'enseignement d'une discipline dans une autre langue doit être appuyé sur des méthodologies et pratiques professionnelles qui utilisent plusieurs types d'alternances : la macro-alternance, la micro-alternance et la méso-alternance ou alternance séquentielle (sous forme de séquences successives).

Le professeur de DdNL devra planifier des séquences pédagogiques, c'est-à-dire, planifier un ensemble de sujets/thèmes organisés afin de travailler le programme annuel dans lequel nous devons reconnaître l'approche conceptuelle, méthodologique et présenter les ressources. C'est le chemin pour le développement des projets interdisciplinaires, approfondissant la connaissance des quatre types de savoirs associés à la compétence interculturelle : savoir, savoir-être, savoir-faire et savoir-apprendre. Les activités doivent être conçues en respectant la présentation de la thématique définie, la durée de la séquence, des objectifs disciplinaires, des objectifs linguistiques et des activités proposées. Et peuvent être conçues avec l'aide d'un professeur de FLE.

Le développement d'un travail collaboratif entre le professeur de FLE et le professeur de la DdNL enseignée est important. La préparation du programme et des cours de DdNL peut être planifiée par les deux professeurs. Ils peuvent anticiper les difficultés des apprenants. Le titre de l'unité didactique peut être présenté aux élèves dans les deux langues. L'alternance des langues doit être présente dans les documents présentés. Un répertoire des mots-clés dans les deux langues, les règles, les définitions, les devoirs, les tests, QCM (questions à choix multiples) doivent être donnés dans les deux langues en alternance avec plusieurs options (question en LM, réponse en LE, par exemple) et sans traduction. Le cours d'une DdNL ne doit pas être une traduction en LE d'un cours de mathématiques en LM, par exemple. II faut qu'il soit conçu comme un cours de DdNL-mathématiques. Le professeur ne traduit pas en LE une consigne ou une explication donnée en LM. Le lexique en LE est préparé et sa compréhension est facilitée en amont, par la distribution des documents à lire, à compléter à la maison, documents vidéo, ... Si cela est nécessaire le document pourra être simplifié à partir d'une révision du professeur de FLE.

Les professeurs de DdNL développent dans leur pratique une conscience linguistique et une compétence discursive dans la matière dont ils sont spécialistes. Ils doivent être sensibles aux particularités linguistico-discursives des matières enseignées en LE. Vollmer, (2006), certifie que la dimension linguistique des activités d'apprentissage est parfois cachée et partiellement implicite et son importance sous-estimée. La formation des enseignants de DdNL comprend également le développement de cette compétence discursive bi/plurilingue (CAUSA, 2016) dans 

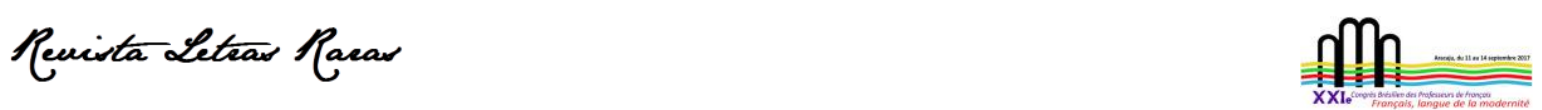

Actes du XXlème Congrès Brésilien des Professeurs De Français dans Édition spéciale de la Revue Letras Raras, 2021 ISSN : 2317-2347 - v. 10, Dossier Spécial (2021)

Todo o conteúdo da RLR está licenciado sob Creative Commons Atribuição 4.0 Internacional

l'enseignement-apprentissage de contenus disciplinaires en langue étrangère, réalisant ainsi une articulation entre le discours commun (de l'usage quotidien de la langue étrangère) et le discours spécialisé (discours de discipline). Cette compétence est construite dans l'articulation entre les documents/supports utilisés et les échanges produits dans le processus interactif. L'enseignement/apprentissage d'une LE et d'une DdNL en LE demande réflexion sur les formes et les fonctionnements discursifs en LE et en LM. Les professeurs développent un travail pédagogique où ils devront observer des documents, identifier les variantes du discours et mettre en place une compétence de médiation (entre les langues, entre les disciplines et entre les discours). Enseigner une DdNL, c'est entrer dans une logique et une méthodologie adaptées à chaque langue.

Les préparations des cours et la formation de ce professeur sont indispensables. Le professeur doit préparer le lexique et les notions du cours en français en amont. Eco (2004) montre que le langage que nous utilisons dans un domaine ne produit pas un lexique et une syntaxe neutre, qui pourraient être employés dans un autre domaine sans préjugé. Les activités mises en place en cours doivent être actionnelles et doivent permettre aux élèves de s'approprier le lexique en LE par le travail en petits groupes, en binômes et avec des ressources variées. Le professeur de DdNL n'est généralement pas un natif : il n'a pas forcément une maîtrise très poussée de la langue étrangère dans laquelle il enseigne. Ce statut sociolinguistique est paradoxalement à l'origine des bons résultats de ce dispositif d'enseignement. Mais nous traiterons cela dans un autre article.

La formation d'un professeur de DdNL doit fournir un « seuil de sécurité » linguistique (GAJO, 2005) et pédagogique afin de le mettre à l'aise dans la langue dans laquelle il enseigne et dans laquelle les contenus disciplinaires sont transmis. Cette sécurité linguistique et pédagogique devra rendre naturel l'emploi de l'alternance codique/séquentielle dans les activités en classe et dans les interactions enseignant/apprenants en tant que stratégie de communication/médiation. Cela est possible par la prise de conscience des différences entre les orientations épistémologiques des disciplines dans les deux langues/cultures, différences qui se reflètent dans les méthodologies et les stratégies d'enseignement-apprentissage comme dans la conception des manuels scolaires. La formation ne doit pas se limiter aux savoirs sur la discipline linguistique et/ou non linguistique de manière cloisonnée. Elle doit prendre en compte la transversalité entre les disciplines proches (linguistiques et non linguistiques); et plus particulièrement pour 

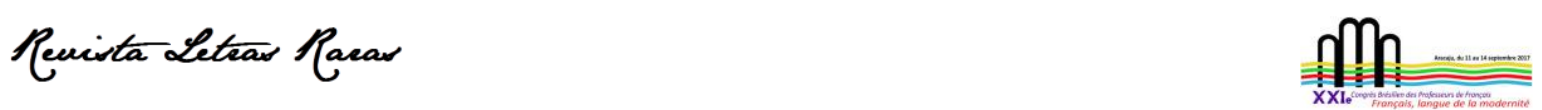

Actes du XXlème Congrès Brésilien des Professeurs De Français dans Édition spéciale de la Revue Letras Raras, 2021 ISSN : 2317-2347 - v. 10, Dossier Spécial (2021)

Todo o conteúdo da RLR está licenciado sob Creative Commons Atribuição 4.0 Internacional

l'enseignement des DdNL. Elle doit se fonder sur les particularités linguistiques et discursives propres à la matière enseignée et à la langue dans laquelle la discipline est enseignée. Les points observés concernant la formation des professeurs de DdNL devront servir de guide pour observer, mener et construire des formations et promouvoir la création d'un travail collaboratif entre le professeur de FLE et le professeur de DdNL.

\section{Considérations finales}

En supposant que l'apprentissage/la formation dépasse l'acquisition de comportements et d'informations, nous soutenons que le travail d'une DdNL et la formation du professeur de DdNL contribuent au développement de la capacité d'auto-organisation, d'auto-évaluation et d'autorégulation de ce professeur. Les professeurs de DdNL doivent avoir conscience de la langue véhiculée, de l'importance du contenu disciplinaire et de la compétence linguistique. II faut acquérir une conscience discursive et linguistique pour le travail de la DdNL. La formation d'un professeur de DdNL devra observer une amélioration des compétences linguistiques, une amélioration des compétences disciplinaires et une amélioration des compétences didactiques et méthodologiques.

Nous croyons que la DdNL est innovante, excitante et stimulante. La théorie apporte des avantages et des gains à la fois pour le professeur et pour l'élève, mais le professeur de DdNL doit être observé en classe. Ce n'est qu'alors qu'il sera possible d'identifier auprès des professeurs les répercussions de cet enseignement sur leur pratique, sur leur identité professionnelle et même sur les stratégies qu'ils peuvent adopter pour faire face aux conflits qui surviennent en classe. II est également à noter que, si le dispositif ne s'inscrit pas dans un projet institutionnel, il n'aura pas des racines profondes permettant sa continuité dans un espace scolaire. Sans parler du travail du professeur de DdNL qui, de ce point de vue, augmente considérablement par rapport à la préparation des cours.

Cette perspective comporte trois éléments: le professeur, l'élève et le contenu. Travailler avec ce type de dispositif peut ouvrir des possibilités aux professeurs de se réinventer. L'élève, à son tour, peut s'ouvrir aux autres, développer de l'empathie, s'immerger dans la diversité et développer des compétences interculturelles. Ce type de travail peut aider l'élève à ouvrir une fenêtre sur le monde. Quant au contenu disciplinaire, serait-il possible de le réduire ou de le 

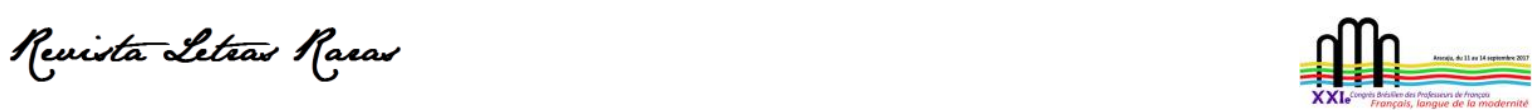

Actes du XXlème Congrès Brésilien des Professeurs De Français dans Édition spéciale de la Revue Letras Raras, 2021 ISSN : 2317-2347 - v. 10, Dossier Spécial (2021)

Todo o conteúdo da RLR está licenciado sob Creative Commons Atribuição 4.0 Internacional

compromettre avec l'utilisation d'une langue étrangère dans les cours ? Et les limites de cela ? II existe d'autres façons de saisir cette perspective. Avec cet article, le premier pas a été franchi, celui de systématiser une littérature très intéressante et pertinente sur le sujet proposé et appartenant à ceux qui prennent des risques dans ce monde du bi-plurilinguisme.

\section{Références}

CAUSA, Mariella. Competênce discursive et enseignement d'une discipline non linguistique : définition, diversification et pratiques formatives, Les Carnets du Cediscor (En ligne), 12/2014, document 6, mis en ligne de 01 mars 2016, consulté le 31 octobre 2016. URL: http://cediscor.revues.org/964

CECR - CONSEIL DE L'EUROPE. Comité de l'Éducation. Un Cadre Européen Commun de Référence pour les Langues: apprendre, enseigner, évaluer. Strasbourg: Didier, 2005.

COSTE, D. Construire des savoirs en plusieurs langues. Les enjeux disciplinaires de l'enseignement bilingue. Association pour le Développement de l'Enseignement Bi/plurilingue, 2003.

COSTE, D. Quelle didactique pour quels contextes ? Enseignement du français au Japon 33. Revue de la Société japonaise de didactique du français, p. 1-14, 2005.

CUQ, J. P.; GRUCA, I. Cours de Didactique du Français Langue Estrangère et Seconde. 4e édition. Grenoble : Presses universitaires de Grenoble, 2017.

DUVERGER, J. L'enseignement en classe bilingue. Hachette Livre, v. 43, n. 15, 2009.

DUVERGER, J. Enseignement Bilingue. « Discipline Non Linguistique » Statut, fonctions, pratiques pédagogiques. France : Institut Français et Délégation générale à la langue française et aux langues de France, 2010.

ECO, Umberto. Os limites da interpretação. São Paulo: Difel, 2004.

GAJO, L. Bilinguisme, immersion et interaction en classe. Paris: Didier. Coll.LAL, 2001.

GAJO, L. Le français langue seconde d'enseignement: choix de modèles, de langues et de disciplines. In: LALLEMENT, F.; MARTINEZ, P.; SPAËT, V. (éds). Français langue d'enseignement, vers une didactique comparative. Le français dans le monde, no especial, janvier, 2005.

LAMBERT, Werner. " Evaluation reactions to spoken language » Journal of Abnormal Psychology 60 Werner Lambert $\mathrm{A}$ study of the roles of attitudes and motivation in second language learning . 1960. http://www.francophonie.org/

MOIRAND, S. Enseigner à communiquer en langue étrangère, Paris, Hachette, (coll. « F»), 1982. 

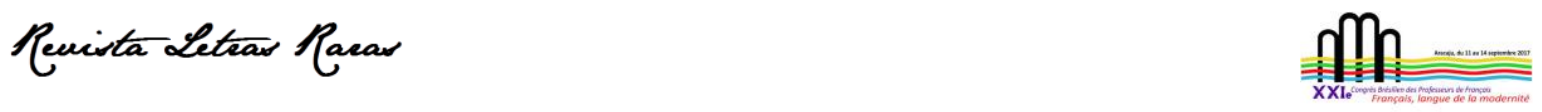

Actes du XXlème Congrès Brésilien des Professeurs De Français dans Édition spéciale de la Revue Letras Raras, 2021 ISSN : 2317-2347 - v. 10, Dossier Spécial (2021)

Todo o conteúdo da RLR está licenciado sob Creative Commons Atribuição 4.0 Internacional

VOLLMER, H. J. Vers un instrument européen commun pour la/les langues de scolarisation. Division des Politiques linguistiques, Strasbourg: Conseil de l'Europe, 2006. Disponible en ligne. http://www.coe.int.

ZABALA, Antoni. A prática educativa, como ensinar. Porto Alegre: ArtMed,1998. 